\title{
High-Performance Thin-Film Composite Membrane with an Ultrathin Spray-Coated Carbon Nanotube Interlayer
}

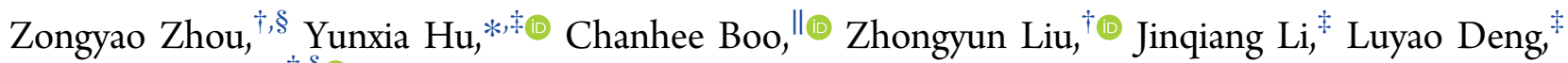
and Xiaochan $\mathrm{An}^{\dagger, \S(0)}$

${ }^{\dagger}$ CAS Key Laboratory of Coastal Environmental Processes and Ecological Remediation, Yantai Institute of Coastal Zone Research, Chinese Academy of Sciences, Yantai 264003, P. R. China

${ }^{\ddagger}$ State Key Laboratory of Separation Membranes and Membrane Processes, National Center for International Research on Membrane Science and Technology, School of Materials Science and Engineering, Tianjin Polytechnic University, Tianjin 300387, P. R. China

${ }^{\S}$ University of Chinese Academy of Sciences, Beijing 100049, P. R. China

"Department of Chemical and Environmental Engineering, Yale University, New Haven, Connecticut 06520-8286, United States

\section{Supporting Information}

ABSTRACT: We fabricated a thin-film composite (TFC) forward osmosis (FO) membrane with an ultrathin spray-coated carbon nanotube (CNT) interlayer. The impact of the CNT interlayer on the polyamide (PA) layer structural properties and transport behavior in FO were investigated. Results indicate that the CNT interlayer provides an interface which enables the formation of a highly permeable and selective PA layer with a large effective surface area for water transport, while inhibiting the formation of a flowerlike PA structure inside the substrate pores. The TFC-FO membrane with the CNT interlayer exhibited a much greater water flux than previously reported for FO membranes, while maintaining comparable salt rejection. Specifically, a membrane perm-selectivity or ratio of water $(A)$ to salt permeability coefficients $(B)\left(A / B\right.$ value) of $39 \mathrm{bar}^{-1}$ was achieved for the TFC-PA-CNT membrane. Implications of the results for the fabrication of highperformance TFC-FO membranes are further discussed.

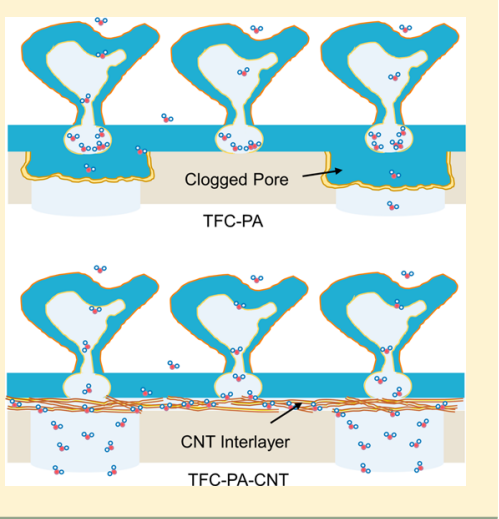

\section{INTRODUCTION}

Osmotically driven membrane processes, such as forward osmosis (FO) and pressure-retarded osmosis (PRO), have been extensively investigated for production of fresh water from a variety of water sources, concentration of aqueous products, and generating power from natural or anthropogenic salinity gradients. ${ }^{1-4}$ Highly selective osmotic membranes that allow fast water transport and maintain low salt permeation are at the core of these emerging technologies. Thin-film composite (TFC) polyamide (PA) membranes with superior selectivity and water permeability provide materials for state-of-the-art osmotic membranes. ${ }^{5-9}$ However, water flux of TFC membranes is significantly constrained by internal concentration polarization (ICP) within the support layer. ${ }^{10}$

Recently, membrane fabrication studies have reported the use of nanostrands, ${ }^{11}$ carbon nanotubes, ${ }^{12}$ or cellulose nanofibers $^{13}$ as an interlayer or sublayer to produce a thin, defect-free PA selective layer to improve water permeability of TFC membranes. Such interlayers have only been applied to fabricate a relatively loose selective layer for nanofiltration membrane for rejection of organic dyes and divalent ions. To date, however, no studies exist on the use of such interlayer or sublayer for the fabrication of dense, highly selective PA layers for osmotic or desalination membranes.

The use of an interlayer material with targeted properties for the fabrication of a selective PA layer for TFC membranes may result in highly permeable and selective layers. The interlayer material can also prevent the interference of a support membrane during the formation of the PA film, thus allowing a wider range of support materials for osmotic membranes with reduced ICP. Moreover, there is a critical need to understand the impact of interlayers on the structural and transport properties of the PA layer to better design and optimize TFCPA osmotic and desalination membranes.

In this study, we spray coat an ultrathin carbon nanotube (CNT) layer on a porous poly(ether sulfone) (PES) support to fabricate a high-performance TFC membrane with a highly permeable and selective polyamide layer. We investigate the top and back surfaces, cross-sectional morphology, and chemical composition of the PA layer formed on the PES support, with and without a CNT interlayer. Water and reverse salt fluxes of the fabricated TFC-PA osmotic membranes were evaluated to determine the membrane perm-selectivity. We also elucidate the impact of the CNT interlayer on the structural and transport properties of the PA layer of the fabricated membrane.

Received: March 26, 2018

Revised: April 11, 2018

Accepted: April 11, 2018

Published: April 11, 2018 

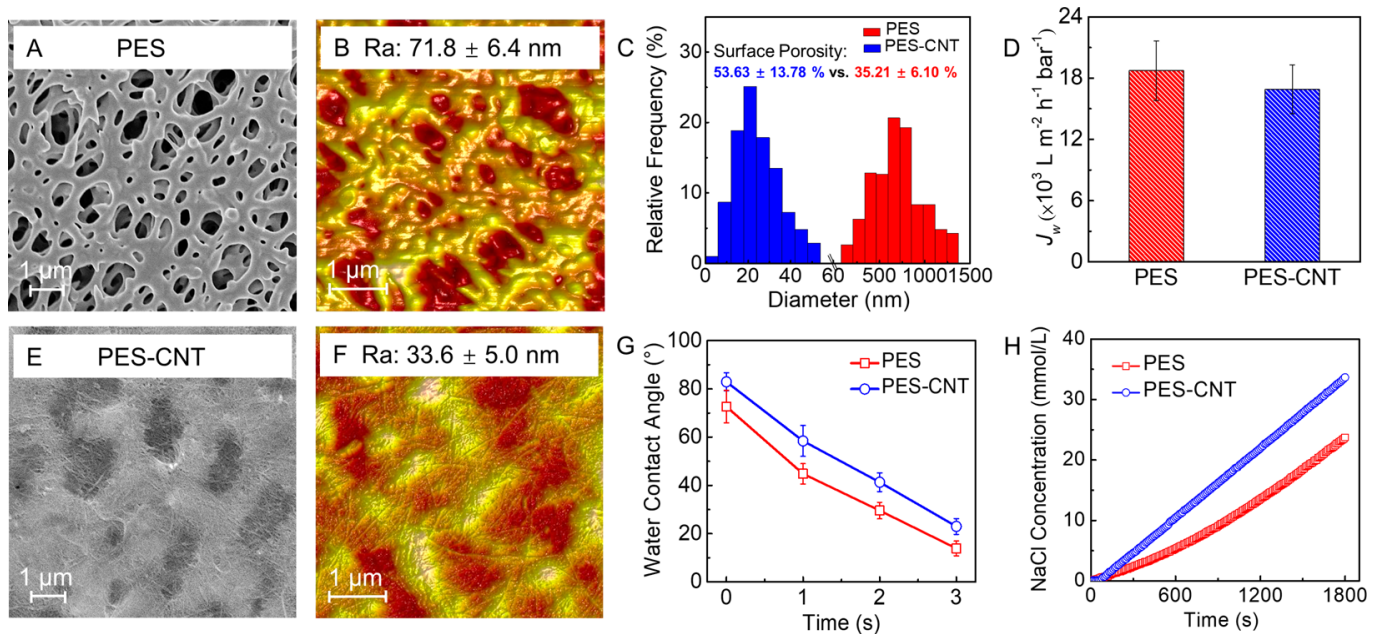

Figure 1. Surface properties of pristine PES and CNT-coated PES membranes (denoted as PES-CNT): (A, E) SEM and (B, F) AFM images, (C) surface porosity and pore size distribution, and (G) water contact angle. Transport properties of pristine PES and PES-CNT membranes: (D) water permeability coefficient determined with dead-end filtration unit at hydraulic pressure of 5 bar and $(\mathrm{H})$ rate of salt diffusion across the membrane as a function of time obtained from diffusion cell experiments.

\section{MATERIALS AND METHODS}

Thin-Film Composite Membrane Fabrication. The fabrication process of the TFC-FO membrane with a CNT interlayer is illustrated in Figure S1. A suspension of single wall CNTs (diameter of $<2 \mathrm{~nm}$, length of 5-30 $\mu \mathrm{m}$, and purity of $>95 \%$ from Aladdin Co., Ltd., Shanghai, China) coated with polydopamine (PDA) was prepared following the reported protocol. $^{12}$ The prepared $5 \mathrm{~mL}$ of the PDA-coated CNT suspension $(0.183 \mathrm{mg} / \mathrm{mL})$ was spray coated on a commercial PES microfiltration membrane with a nominal pore size of 0.22 $\mu \mathrm{m}$ (Yibo Co., Ltd., Zhejiang, China) using an airbrush (LPH50-S9, Anest Iwata, Japan) at $0.1 \mathrm{MPa}$ and at a $30 \mathrm{~cm}$ distance from the membrane at room temperature under $20 \mathrm{RH} \%$ humidity. After drying at $60^{\circ} \mathrm{C}$ for $0.5 \mathrm{~h}$, the CNT-coated PES membrane was prewetted in DI water for $0.5 \mathrm{~h}$. The polyamide (PA) selective layer was formed on the CNT interlayer via interfacial polymerization (IP) of $m$-phenylenediamine (MPD, $3.4 \mathrm{wt} \%$ ) in water and trimesoyl chloride (TMC, $0.15 \mathrm{wt} \%$ ) in hexane following our previous work. ${ }^{14}$ More details on the fabrication of the TFC-FO membrane are provided in the Supporting Information. The photographs of the prepared TFC-FO membranes are shown in Figure S2.

Membrane Characterization. Scanning electron microscopy (SEM, S-4800, Hitachi, Japan) was used to observe the surface and cross-sectional morphologies of the prepared TFCFO membranes. Membrane samples were coated with platinum $(\mathrm{Pt})$ for $100 \mathrm{~s}$ utilizing an EMITECH SC7620 sputter coater. X-ray photoelectron spectroscopy (XPS, Thermo Esca Lab $250 \mathrm{Xi}$, USA) was employed to determine the cross-linking degree of the PA film by analyzing the elemental composition of the top and back surfaces of the PA layer following the reported method. ${ }^{15}$ Atomic force microscopy (AFM, MultiMode 8, Veeco, US) was used to investigate the membrane surface roughness in tapping mode at a scan rate of $1 \mathrm{~Hz}$ using TESP tips (Sharp silicon probe, $42 \mathrm{~N} / \mathrm{m}, 320 \mathrm{kHz}$, tip radius 8 $\mathrm{nm}$, Bruker, USA). AFM images were processed using Nanoscope Analysis software to determine membrane surface roughness. The water contact angles (WCA) were measured using an optical instrument (OCA20, Data Physics, Germany) at room temperature. A $3.0 \mu \mathrm{L}$ DI water droplet was dropped from a microsyringe on the membrane surface, and images of dynamic droplet shape were recorded. The water contact angle was determined from the recorded images using the automated Drop Shape Analysis software (SCA20 Version 2). The size and distribution of pores were evaluated by analyzing SEM images of the synthesized PA film using the Nano Measurer software. The surface porosity was determined using the ImageJ software. At least nine SEM images from three individual samples were analyzed to obtain an average value.

Membrane Performance Evaluation. Water flux of the TFC-PA and TFC-PA-CNT membranes were measured in a cross-flow lab-scale FO system (Figure S3). The feed and draw solutions were $\mathrm{DI}$ water and $1 \mathrm{M} \mathrm{NaCl}$ solution, respectively, at a temperature of $25.0 \pm 0.5{ }^{\circ} \mathrm{C}$. The membranes were tested under both PRO (active layer facing draw solution) and FO (active layer facing feed solution) modes. The water permeability coefficient $(A)$, salt permeability coefficient $(B)$, and structural parameter $(S)$ of the TFC-FO membrane were determined according to the previously developed $\mathrm{FO}$ characterization method. ${ }^{16}$ More details on the TFC-FO membrane performance evaluation are provided in the Supporting Information.

A lab-scale diffusion cell (Figure S4) was used to measure the rate of salt $(\mathrm{NaCl})$ diffusion across the pristine PES and CNTcoated PES (denoted as PES-CNT) membranes. One chamber was filled with $35 \mathrm{~mL}$ of DI water and another with $35 \mathrm{~mL}$ of 1 $\mathrm{M} \mathrm{NaCl}$ solution. The membrane was placed in between two chambers. The solution conductivity in the DI water chamber was monitored using a conductivity meter (CON2700, Eutech, USA). The rate of salt diffusion over time was determined based on a calibration curve of salt concentration versus solution conductivity.

\section{RESULTS AND DISCUSSION}

Impact of CNT Interlayer on Porous Support Properties. Spray coating allows for the deposition of an ultrathin CNT film layer on the porous substrate. Surface properties of the CNT-coated PES membrane (PES-CNT) including morphology, pore size, porosity, roughness, and hydrophilicity were investigated. Such substrate surface properties have been reported to influence the formation of a PA selective layer 

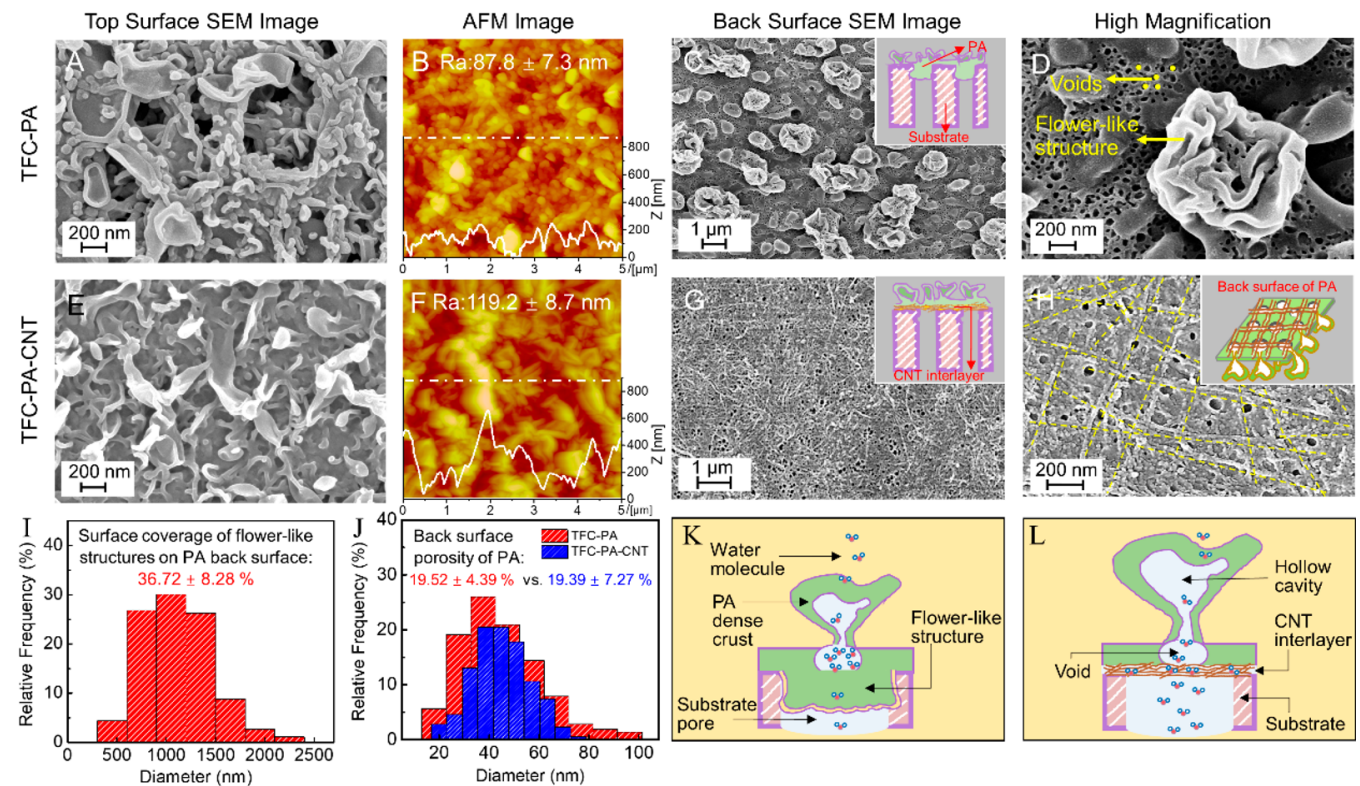

Figure 2. (A, B, E, and F) Morphologies of top surface and (C, D, G, and H) back surface of PA layer formed on pristine PES substrate (TFC-PA membrane) and CNT-coated PES substrate (TFC-PA-CNT membrane). (I) Size distribution of flower-like structure developed on back surface of PA layer determined from SEM image shown in panel C. (J) Surface porosity and surface pore size distribution determined from SEM images shown in panels $\mathrm{C}$ and $\mathrm{D}$. Proposed cross-sectional structures of PA layer formed on (K) pristine PES support and (L) CNT-coated PES support. A, C, D, $\mathrm{E}, \mathrm{G}$, and $\mathrm{H}$ are SEM images, and $\mathrm{B}$ and $\mathrm{F}$ are AFM images. White dashed line of the AFM images (B, F) marks the location where the crosssectional height profiles were obtained.

during interfacial polymerization. ${ }^{17-20}$ As Figure 1E shows, the PES-CNT membrane has a continuous and porous CNT network structure covering the underlying PES substrate (Figure 1A). The large pores of PES substrate underneath the CNT network are discernible from SEM and AFM images of the PES-CNT membrane (Figure $1 \mathrm{E}$ and F) due to the ultrathin CNT layer. The statistical analysis based on the SEM images (Figure $1 \mathrm{~A}$ and $\mathrm{E}$ ) indicates that the average surface pore size substantially decreased from $702 \pm 36$ to $21 \pm 7 \mathrm{~nm}$ for the pristine PES and PES-CNT membranes, respectively, after CNT spray coating. Notably, the surface porosity increased upon spray coating of the CNT interlayer, likely due to the porous structure of the fibrous $\mathrm{CNT}$ interlayer (Figure 1C). The CNT interlayer provides a smoother surface as evidenced by a lower roughness of the PES-CNT membrane compared to the pristine PES substrate (Figure $1 \mathrm{~B}$ and F). Water contact angles of the PES-CNT and pristine PES membranes were measured to be comparable, suggesting that these membranes have similar surface hydrophilicity (Figure $1 \mathrm{G}$ and Figure S5).

Water permeability coefficients of the pristine PES and PESCNT membranes were comparable $\left(\sim 17,000-18,000 \mathrm{~L} \mathrm{~m}^{-2}\right.$ $\mathrm{h}^{-1} \mathrm{bar}^{-1}$, Figure 1D) and much higher than that of ultrafiltration membranes (approximately $300-800 \mathrm{~L} \mathrm{~m}^{-2} \mathrm{~h}^{-1}$ bar $^{-1}$ ) widely used as a TFC osmotic membrane substrate. ${ }^{21,22}$ It is worth noting that the rate of salt diffusion across the PESCNT membrane is faster than that across the pristine PES membrane (Figure $1 \mathrm{H}$ ), implying a reduced resistance to salt transport through the PES-CNT substrate, and it can be beneficial to have a TFC osmotic membrane with a lower extent of ICP within the support layer.

Impact of CNT Interlayer on Polyamide Layer Structure. The PA layer of the TFC-PA-CNT membrane was rich in characteristic ridge-and-valley and leaf-like structures with a higher surface roughness (Figure 2E and F) than that of the TFC-PA membrane which had a typical "nodular" feature with a few leaf-like structures (Figure 2A). This observation agrees with the literature results ${ }^{23}$ that a rougher PA layer is formed on a relatively dense substrate because a higher interfacial instability induced by heat generated from fast IP reaction between MPD and TMC provides additional interfacial area to form PA sublayers. Interestingly, the back surface of the PA layer of the TFC-PA membrane was abundant in flower-like structures that covered much of PES substrate surface porosity (Figure 2C and D and Figure S7). The size of such a flower-like structure is remarkedly larger than the substrate pores. We hypothesize that these flower-like structures are the PA film formed inside the pores of a PES support (pore size of $0.22 \mu \mathrm{m}$ ). The observed large flower-like PA structure may be created due to the diffusion of MPD across the sponge-like interpores of the PES support to react with TMC, forming PA pillars with a big head as illustrated in Figure $2 \mathrm{~K}$. In contrast, nanofibrous CNT bundles are observed on the back surface of a PA layer of the TFC-PA-CNT membrane (Figure $2 \mathrm{G}$ and $\mathrm{H}$ and Figure S7). Numerous voids that provide comparable pore size and porosity are present on the back surface of the PA layers regardless of the CNT interlayer (Figure 2J). These voids have been reported to form on the PA layer facing the MPD aqueous solution and serve as a tunnel for the diffusion of MPD to a newly formed PA interface during the IP process. ${ }^{24}$ These voids also provide a channel for water transport through the PA selective layer. ${ }^{24}$ The mechanism responsible for the formation of such voids and their impacts on PA layer transport properties require further investigation.

The cross-sectional SEM images of the TFC-PA-CNT membrane present a clear interface between the PA film and the CNT interlayer (Figure S9), confirming the PA layer formation on top of the CNT interlayer as described in Figure $2 \mathrm{~L}$. In contrast, there is no clear interface between the PA film 
and the PES substrate of the TFC-PA membrane (Figure S10), likely indicating that the PA layer is developed from inside the PES substrate pores. Interestingly, numerous hollow structure protuberances and approximately $25 \mathrm{~nm}$ thick crusts are observed from PA layers of both the TFC-PA and PFC-PACNT membranes (Figures S9 and S10), which further confirms an intrinsic PA thickness of around $25 \mathrm{~nm}$, very close to the reported value of $20 \mathrm{~nm}$ thickness of PA selective layer created by self-limiting IP process. ${ }^{11,25}$

Results from XPS analysis indicate that the cross-linking degree of the PA layer formed on the PES substrate is markedly different for the top surface $(17.39 \%)$ and the bottom surface $(84.36 \%)$, which is in agreement with the results from previous studies (Figure S11 and Table S1). ${ }^{26,27}$ The top surface of the $\mathrm{PA}$ layer on the CNT interlayer exhibited a higher cross-linking degree than that on the PES substrate. Unfortunately, we were not able to determine the cross-linking degree of the back surface of the PA film on the CNT layer due to the interference of $\mathrm{C}, \mathrm{O}$, and $\mathrm{N}$ signals from the PDA and CNT.

Membrane Transport Properties. Water flux of the TFCPA-CNT membrane was dramatically improved by $610 \%$ to $38.0 \pm 2.0 \mathrm{~L} \mathrm{~m}^{-2} \mathrm{~h}^{-1}$ in PRO mode with $1 \mathrm{M} \mathrm{NaCl}$ as a draw solution and DI water as a feed solution compared to the TFCPA membrane without the CNT interlayer (Figure 3A). The
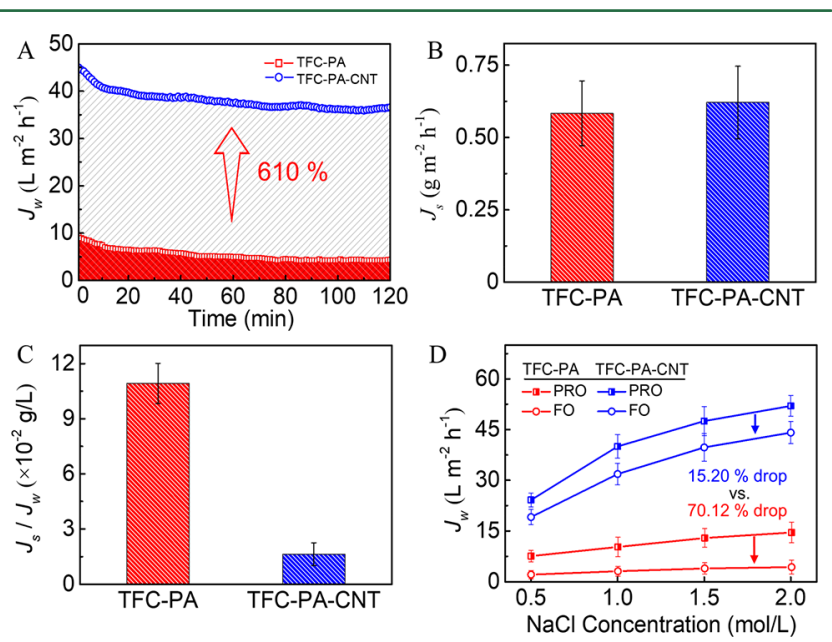

Figure 3. (A) Water flux, $J_{w}$, (B) reverse salt flux, $J_{s}$, and (C) $J_{s} / J_{w}$ value of the TFC-PA and TFC-PA-CNT membranes obtained in PRO mode using $1 \mathrm{M} \mathrm{NaCl}$ as a draw solution and DI water as a feed solution. (D) Water fluxes of TFC-PA and TFC-PA-CNT membranes determined in $\mathrm{FO}$ and PRO modes with various draw solution $\mathrm{NaCl}$ concentrations and DI water as a feed solution.

obtained water flux of the TFC-PA-CNT membrane is $2-2.5$ times higher than commercial TFC-FO membranes from Hydration Technology Innovation (HTI) evaluated under similar conditions. ${ }^{14,28}$ We further observed that the reverse salt fluxes of the TFC-PA-CNT and TFC-PA membranes were almost 10-50-folds lower (Figure 3B) than the values reported for commercial TFC-FO membranes $\left(5-35 \mathrm{~g} \mathrm{~m}^{-2} \mathrm{~h}^{-1}\right){ }^{28,29}$ The specific salt flux $\left(J_{s} / J_{w}\right)$ of the TFC-PA-CNT membrane $\left(\sim 1.6 \times 10^{-2} \mathrm{~g} / \mathrm{L}\right.$ in Figure $\left.3 \mathrm{C}\right)$ is approximately 7 -fold lower than that of the TFC-PA membrane, which highlights an excellent perm-selectivity of the PA layer formed on the CNT interlayer.

The water permeability coefficient $(A)$ of the TFC-PA-CNT membrane is $2.0 \pm 0.2 \mathrm{~L} \mathrm{~m}^{-2} \mathrm{~h}^{-1} \mathrm{bar}^{-1}$ (Table S2), which is about 7 -fold higher compared to that of the TFC-PA membrane and higher than most of hand-made and commercial TFC-FO membranes found in literatures. ${ }^{30-33}$ The salt permeability coefficient $(B)$ of the TFC-PA-CNT membrane is $0.05 \pm 0.01 \mathrm{~L} \mathrm{~m}^{-2} \mathrm{~h}^{-1}$, comparable to that of the TFC-PA membrane, far below than the values reported for other handmade and commercial TFC-FO membranes $\left(1.0-1.7 \mathrm{~L} \mathrm{~m}^{-2}\right.$ $\left.\mathrm{h}^{-1}\right){ }^{16,28}$ Importantly, the TFC-PA-CNT membrane achieves the highest ratio of water to salt permeability coefficients $(A / B)$ of $39 \mathrm{bar}^{-1}$ among the currently reported TFC-FO membranes, ${ }^{32,34}$ further demonstrating an excellent permselectivity of the TFC-PA-CNT membrane.

The observed high perm-selectivity of the TFC-FO membrane with the CNT interlayer can be ascribed to the following two major reasons: (1) The CNT interlayer provides a favorable interface to form the highly permeable and selective PA layer with large effective surface area for water transport. (2) The CNT interlayer inhibits the formation of the flower-like PA film inside the pores of PES substrate, which reduces the resistance to water transport. We also speculate that the CNT interlayer develops a channel for water transport from the PA film formed on the non-porous domain of PES substrate to neighboring pores as illustrated in Figure S12. Further studies are required to verify this proposed mechanism.

The structural parameter, $S$, is a direct measure of the performance limiting phenomenon of internal concentration polarization (ICP) of TFC osmotic membranes. ${ }^{16,35}$ The $S$ value of the TFC-PA-CNT membrane is measured to be significantly lower compared to that of the TFC-PA membrane (Table S2), which is contradicted to the expectation that a lower support layer resistance to salt diffusion translates to a smaller $S$ value because the support of the TFC-PA-CNT membrane has an additional resistance attributed to the CNT interlayer. We thus propose that the hollow structure of the PA film may influence the $S$ value of the TFC-FO membrane by increasing the effective distance for salt transport. The ratio of water flux measured in $\mathrm{FO}$ and PRO modes $\left(J_{w, F O} / J_{w, P R O}\right)$ represents another indicator of the extent of ICP, with a higher value indicating a lower extent of ICP. ${ }^{10}$ As shown in Figure $3 \mathrm{D}$, the TFC-PA-CNT membrane has $J_{w, F O} / J_{w}$, PRO values in the range of $0.79-0.85$ with various $\mathrm{NaCl}$ draw solution concentrations, which are about $2-3$ folds higher than the TFC-PA membrane, implying a significantly reduced ICP within the support layer. We further evaluated the water flux in a long-term operation to confirm the stability of PA selective layer formed on the CNT layer. The TFC-PA-CNT membrane exhibited a stable water flux for a $30-\mathrm{h}$ experiment in PRO mode, demonstrating a robust PA layer perm-selectivity (Figure S13).

Implications. We have fabricated a high-performance TFC osmotic membrane with an ultrathin spray-coated CNT interlayer. The use of an ultrathin nanofibrous interlayer on a porous substrate allows for the fabrication of a highly permeable and selective polyamide layer for TFC membranes. Additionally, an ultrathin nanofibrous interlayer serves as a support with a low thickness and a low tortuosity, allowing the osmotic membranes to produce high water flux with reduced internal concentration polarization. Designing such an ultrathin interlayer provides a great potential to fabricate high performance TFC desalination membranes, including reverse osmosis and nanofiltration membranes. 


\section{ASSOCIATED CONTENT}

\section{S Supporting Information}

The Supporting Information is available free of charge on the ACS Publications website at DOI: 10.1021/acs.estlett.8b00169.

Details on preparation of PDA-coated CNT suspension, fabrication of PES-CNT and TFC-PA-CNT membranes, and characterization of TFC-FO membrane transport properties. Schematic describing fabrication of TFC-PACNT membrane (Figure S1). Digital photos of pristine PES substrate, PES-CNT membrane, and TFC-PA-CNT membrane (Figure S2). Schematic drawing of cross-flow lab-scale FO system (Figure S3) and lab-scale diffusion cell (Figure S4). Digital photos of water contact angle on PES substrate and PES-CNT membrane (Figure S5). SEM images of top (Figure S6) and back PA surfaces (Figure S7) of TFC-PA membrane and TFC-PA-CNT membrane. SEM images of top and back PA surface morphologies of the TFC-PA membrane (Figure S8). Cross-sectional SEM and schematically visualized images of TFC-PA-CNT membrane (Figure S9). Cross-sectional SEM and schematically visualized images of TFCPA membrane (Figure S10). Cross-linking degrees of PA layers (Figure S11). 3D diagrams of TFC-PA and TFCPA-CNT membranes (Figure S12). Long-term water flux of TFC-PA-CNT membrane evaluated in PRO mode (Figure S13). XPS results of PA layer (Table S1). Water permeability coefficient $(A)$, solute permeability coefficient $(B)$, and structural parameter $(S)$ of the fabricated TFC-FO membranes (Table S2). (PDF)

\section{AUTHOR INFORMATION}

\section{Corresponding Author}

*Tel: +86-22-83955129. E-mail: yunxiahu@yic.ac.cn.

\section{ORCID}

Yunxia Hu: 0000-0003-4710-6542

Chanhee Boo: 0000-0003-4595-9963

Zhongyun Liu: 0000-0002-9078-0404

Xiaochan An: 0000-0002-4765-3178

Notes

The authors declare no competing financial interest.

\section{ACKNOWLEDGMENTS}

The authors gratefully acknowledge the funding support from National Natural Science Foundation of China (No. 21476249, No. 51708408), Chang-jiang Scholars and Innovative Research Team in the University of Ministry of Education, China (No. IRT-17R80), and The Science and Technology Plans of Tianjin (No. 17PTSYJC00060).

\section{REFERENCES}

(1) Shannon, M. A.; Bohn, P. W.; Elimelech, M.; Georgiadis, J. G.; Marinas, B. J.; Mayes, A. M. Science and technology for water purification in the coming decades. Nature 2008, 452 (7185), 301-10.

(2) Xie, M.; Nghiem, L. D.; Price, W. E.; Elimelech, M. Toward Resource Recovery from Wastewater: Extraction of Phosphorus from Digested Sludge Using a Hybrid Forward Osmosis-Membrane Distillation Process. Environ. Sci. Technol. Lett. 2014, 1 (2), 191-195.

(3) Hoover, L. A.; Phillip, W. A.; Tiraferri, A.; Yip, N. Y.; Elimelech, M. Forward with osmosis: emerging applications for greater sustainability. Environ. Sci. Technol. 2011, 45 (23), 9824-30.

(4) Gu, Y.; Chen, L.; Ng, J.-W.; Lee, C.; Chang, V. W. C.; Tang, C. Y. Development of anaerobic osmotic membrane bioreactor for low- strength wastewater treatment at mesophilic condition. J. Membr. Sci. 2015, 490, 197-208.

(5) Tiraferri, A.; Kang, Y.; Giannelis, E. P.; Elimelech, M. Superhydrophilic thin-film composite forward osmosis membranes for organic fouling control: fouling behavior and antifouling mechanisms. Environ. Sci. Technol. 2012, 46 (20), 11135-44.

(6) Zhao, S.; Zou, L.; Tang, C. Y.; Mulcahy, D. Recent developments in forward osmosis: Opportunities and challenges. J. Membr. Sci. 2012, 396, 1-21.

(7) Yip, N. Y.; Tiraferri, A.; Phillip, W. A.; Schiffman, J. D.; Elimelech, M. High performance thin-film composite forward osmosis membrane. Environ. Sci. Technol. 2010, 44 (10), 3812-8.

(8) Elimelech, M.; Phillip, W. A. The Future of Seawater Desalination: Energy, Technology, and the Environment. Science 2011, 333 (6043), 712-717.

(9) Lu, X.; Nejati, S.; Choo, Y.; Osuji, C. O.; Ma, J.; Elimelech, M. Elements Provide a Clue: Nanoscale Characterization of Thin-Film Composite Polyamide Membranes. ACS Appl. Mater. Interfaces 2015, 7 (31), 16917-22.

(10) Song, X.; Liu, Z.; Sun, D. D. Nano gives the answer: breaking the bottleneck of internal concentration polarization with a nanofiber composite forward osmosis membrane for a high water production rate. Adv. Mater. 2011, 23 (29), 3256-60.

(11) Karan, S.; Jiang, Z.; Livingston, A. G. Sub-10 nm polyamide nanofilms with ultrafast solvent transport for molecular separation. Science 2015, 348 (6241), 1347.

(12) Zhu, Y.; Xie, W.; Gao, S.; Zhang, F.; Zhang, W.; Liu, Z.; Jin, J. Single-Walled Carbon Nanotube Film Supported Nanofiltration Membrane with a Nearly $10 \mathrm{~nm}$ Thick Polyamide Selective Layer for High-Flux and High-Rejection Desalination. Small 2016, 12 (36), 5034-5041.

(13) Wang, J. J.; Yang, H. C.; Wu, M. B.; Zhang, X.; Xu, Z.-K. Nanofiltration membranes with cellulose nanocrystals as an interlayer for unprecedented performance. J. Mater. Chem. A 2017, 5 (31), 16289-16295.

(14) Liu, Z.; Hu, Y. Sustainable Antibiofouling Properties of Thin Film Composite Forward Osmosis Membrane with Rechargeable Silver Nanoparticles Loading. ACS Appl. Mater. Interfaces 2016, 8 (33), 21666-73.

(15) Khorshidi, B.; Thundat, T.; Fleck, B. A.; Sadrzadeh, M. A Novel Approach Toward Fabrication of High Performance Thin Film Composite Polyamide Membranes. Sci. Rep. 2016, 6, 22069.

(16) Tiraferri, A.; Yip, N. Y.; Straub, A. P.; Romero-Vargas Castrillon, S.; Elimelech, M. A method for the simultaneous determination of transport and structural parameters of forward osmosis membranes. J. Membr. Sci. 2013, 444, 523-538.

(17) Lu, X.; Arias Chavez, L. H.; Romero-Vargas Castrillon, S.; Ma, J.; Elimelech, M. Influence of active layer and support layer surface structures on organic fouling propensity of thin-film composite forward osmosis membranes. Environ. Sci. Technol. 2015, 49 (3), $1436-44$.

(18) Ghosh, A. K.; Hoek, E. M. V. Impacts of support membrane structure and chemistry on polyamide-polysulfone interfacial composite membranes. J. Membr. Sci. 2009, 336 (1-2), 140-148.

(19) Fathizadeh, M.; Aroujalian, A.; Raisi, A. Effect of lag time in interfacial polymerization on polyamide composite membrane with different hydrophilic sub layers. Desalination 2012, 284, 32-41.

(20) Jimenez-Solomon, M. F.; Gorgojo, P.; Munoz-Ibanez, M.; Livingston, A. G. Beneath the surface: Influence of supports on thin film composite membranes by interfacial polymerization for organic solvent nanofiltration. J. Membr. Sci. 2013, 448, 102-113.

(21) Xu, J.; Yao, P.; Li, X.; He, F. Synthesis and characterization of water-soluble and conducting sulfonated polyaniline/para-phenylenediamine-functionalized multi-walled carbon nanotubes nanocomposite. Mater. Sci. Eng., B 2008, 151 (3), 210-219.

(22) Mokhtari, S.; Rahimpour, A.; Shamsabadi, A. A.; Habibzadeh, S.; Soroush, M. Enhancing performance and surface antifouling properties of polysulfone ultrafiltration membranes with salicylate-alumoxane nanoparticles. Appl. Surf. Sci. 2017, 393, 93-102. 
(23) Tian, M.; Qiu, C. Q.; Liao, Y.; Chou, S. R.; Wang, R. Preparation of polyamide thin film composite forward osmosis membranes using electrospun polyvinylidene fluoride (PVDF) nanofibers as substrates. Sep. Purif. Technol. 2013, 118, 727-736.

(24) Pacheco, F.; Sougrat, R.; Reinhard, M.; Leckie, J. O.; Pinnau, I. $3 \mathrm{D}$ visualization of the internal nanostructure of polyamide thin films in RO membranes. J. Membr. Sci. 2016, 501, 33-44.

(25) Yan, H.; Miao, X.; Xu, J.; Pan, G.; Zhang, Y.; Shi, Y.; Guo, M.; $\mathrm{Liu}, \mathrm{Y}$. The porous structure of the fully-aromatic polyamide film in reverse osmosis membranes. J. Membr. Sci. 2015, 475, 504-510.

(26) Gohil, J. M.; Ray, P. A review on semi-aromatic polyamide TFC membranes prepared by interfacial polymerization: Potential for water treatment and desalination. Sep. Purif. Technol. 2017, 181, 159-182.

(27) Ahmad, A.; Ooi, B. Properties-performance of thin film composites membrane: study on trimesoyl chloride content and polymerization time. J. Membr. Sci. 2005, 255 (1-2), 67-77.

(28) Ren, J.; McCutcheon, J. R. A new commercial thin film composite membrane for forward osmosis. Desalination 2014, 343, 187-193.

(29) Wang, Y.-N.; Goh, K.; Li, X.; Setiawan, L.; Wang, R. Membranes and processes for forward osmosis-based desalination: Recent advances and future prospects. Desalination 2018, 434, 81-89.

(30) Duong, P. H.; Chisca, S.; Hong, P. Y.; Cheng, H.; Nunes, S. P.; Chung, T. S. Hydroxyl functionalized polytriazole-co-polyoxadiazole as substrates for forward osmosis membranes. ACS Appl. Mater. Interfaces 2015, 7 (7), 3960-73.

(31) Xiao, P.; Nghiem, L. D.; Yin, Y.; Li, X.-M.; Zhang, M.; Chen, G.; Song, J.; He, T. A sacrificial-layer approach to fabricate polysulfone support for forward osmosis thin-film composite membranes with reduced internal concentration polarisation. J. Membr. Sci. 2015, 481, $106-114$.

(32) Xu, L.; Xu, J.; Shan, B.; Wang, X.; Gao, C. Novel thin-film composite membranes via manipulating the synergistic interaction of dopamine and $\mathrm{m}$-phenylenediamine for highly efficient forward osmosis desalination. J. Mater. Chem. A 2017, 5 (17), 7920-7932.

(33) Xie, M.; Bar-Zeev, E.; Hashmi, S. M.; Nghiem, L. D.; Elimelech, M. Role of Reverse Divalent Cation Diffusion in Forward Osmosis Biofouling. Environ. Sci. Technol. 2015, 49 (22), 13222-9.

(34) Yip, N. Y.; Elimelech, M. Performance limiting effects in power generation from salinity gradients by pressure retarded osmosis. Environ. Sci. Technol. 2011, 45 (23), 10273-82.

(35) Shaffer, D. L.; Werber, J. R.; Jaramillo, H.; Lin, S.; Elimelech, M. Forward osmosis: Where are we now? Desalination 2015, 356, 271284. 